\title{
BMJ Open Association between psychological distress and dietary intake among evacuees after the Great East Japan Earthquake in a cross-sectional study: the Fukushima Health Management Survey
}

\author{
Mayu Uemura, ${ }^{1}$ Tetsuya Ohira, ${ }^{1,2}$ Seiji Yasumura, ${ }^{2,3}$ Akira Otsuru, ${ }^{2,4}$ \\ Masaharu Maeda, ${ }^{2,5}$ Mayumi Harigane, ${ }^{2}$ Naoko Horikoshi, ${ }^{2}$ Yuriko Suzuki, ${ }^{6}$ \\ Hirooki Yabe, ${ }^{2,7}$ Hideto Takahashi, ${ }^{2}$ Masato Nagai, ${ }^{1,2}$ Hironori Nakano, ${ }^{1,2}$ \\ Wen Zhang, ${ }^{1}$ Mayumi Hirosaki, ${ }^{1}$ Masafumi Abe, ${ }^{2}$ for the Fukushima Health \\ Management Survey Group
}

To cite: Uemura M, Ohira T, Yasumura S, et al. Association between psychological distress and dietary intake among evacuees after the Great East Japan Earthquake in a crosssectional study: the Fukushima Health Management Survey. BMJ Open 2016;6:e011534. doi:10.1136/bmjopen-2016011534

- Prepublication history and additional material is available. To view please visit the journal (http://dx.doi.org/ 10.1136/bmjopen-2016011534).

Received 16 February 2016 Revised 28 May 2016 Accepted 1 June 2016

CrossMark

For numbered affiliations see end of article.

Correspondence to Dr Mayu Uemura; mayu-u@fmu.ac.jp

\section{ABSTRACT}

Objective: Psychological distress is generally associated with poor dietary intake, but this has never been investigated among residents after a major disaster. We attempted to reveal the associations between dietary intake and non-specific mental health distress as well as traumatic symptoms among evacuees after the Great East Japan Earthquake of 2011.

Methods: In this cross-sectional analysis of 63047 evacuees (27 901 men, 35146 women) who responded to The Fukushima Health Management Survey in 2012, non-specific mental health distress was assessed using the Kessler-6 (K6) scale, while traumatic symptoms were evaluated using the Posttraumatic Stress Disorder (PTSD) Checklist-StressorSpecific Version (PCL-S). The outcome was 'low frequency'-meaning a daily consumption in the 25th centile or less according to the food frequency questionnaire (FFQ) — of 19 targeted food items. Logistic regression analysis was used to estimate ORs and $95 \%$ Cls adjusted for demographic, lifestylerelated and disaster-related factors.

Results: Of the participants, $14.7 \%$ suffered nonspecific mental health distress, and $21.2 \%$ exhibited traumatic symptoms. Multivariable adjusted logistic regression analysis showed that the former were likely to have a low intake frequency of certain foods, such as rice and bread, fish, meat, vegetables or fruit (non-juice), soya bean products, milk, and yogurt or lactobacillus drinks; the latter were also likely to have a low intake frequency of certain foods, including rice and bread, fish, meat, vegetables (non-juice), milk and yogurt or lactobacillus drinks, but conversely consumed vegetable and fruit juices more often. These associations between dietary intake and non-specific mental health distress, as well as traumatic symptoms, were predominantly observed in women.

\section{Strengths and limitations of this study}

- We examined the association between psychological distress and dietary intake among evacuees of the number of more than 60000 after the Great East Japan Earthquake in a situation where the associations between psychological distress and food intake have never been investigated after major disasters, although major disasters could lead to psychological distress.

- A limitation of this study was an overall low response rate $(40.7 \%)$, and a relatively high percentage of missing observations under the category of psychological distress $(10.3 \%)$. It should be noted that participants in the present study were not always representative of all the residents in Fukushima, and this could have enhanced or weakened the association between psychological distress and dietary intake.

- We could not assess the associations between psychological distress and modern Japanese food, such as processed food, fast food, ready-to-eat foods or recently introduced Western food, as we used a food frequency questionnaire (FFQ) of 19 items, in which some food items that might have been consumed by evacuees with higher probability were not included.

- In addition, information on portion size was not included on the FFQ, which prevented us from calculating the quantity of each food consumed and the participants' dietary nutrient intake.

Conclusions: Psychological distress after the Great East Japan Earthquake among evacuees was associated with a low intake frequency of certain foods, and the association was predominantly observed in women. 


\section{INTRODUCTION}

The Great East Japan Earthquake on 11 March 2011 and the subsequent gigantic tsunami and nuclear incident at Fukushima Daiichi Nuclear Power Plant triggered physical ${ }^{1-3}$ and mental health disorders ${ }^{4}$ in evacuees formerly residing in Fukushima Prefecture. In response to the evacuees' concerns surrounding the disaster and ensuing accidents, the Fukushima Health Management Survey was conducted to investigate longterm effects of low-dose radiation exposure, the evacuees' experiences of disaster-related events and their dramatically altered lifestyle. ${ }^{5}$

These experiences and prolonged unsettled lifestyle have caused a great deal of mental health problems among evacuees, such as anxiety, stress and traumatic symptoms. Prevalences of these have been of serious concern; for example, the prevalence of non-specific mental health distress among evacuees was above average for Japan. ${ }^{4}{ }^{6}$ Furthermore, the prevalence of non-specific mental health distress and traumatic symptoms was higher among evacuees in Fukushima Prefecture than among evacuees in other disaster areas like Miyagi and Iwate Prefectures. ${ }^{78}$

In addition, disaster-related factors like poor living arrangements and decreased income most likely made the lives of evacuees even more difficult. Poor diet in particular has been found to be associated with the incidence of physical diseases and conditions. Psychological distress and poor dietary intake are known to be associated: for example, people with depression tend not to consume fresh foods. ${ }^{9}$ It is thus easy to imagine how mental health problems might be aggravated by poor dietary and nutritional intake. ${ }^{9} 10$

Given the close link between mental health and food intake alluded to above, we believe that support from a healthy diet is a truly essential aspect of alleviating the physical and mental health problems of evacuees. However, these associations between mental health problems and dietary intake have never been investigated in the context of evacuees of major disasters.

The dietary problems of evacuees with mental health problems must be clarified in order to provide them with appropriate healthcare. We therefore examined the association of psychological distress-that is, non-specific mental health distress and traumatic symptoms-with dietary intake among evacuees after the Great East Japan Earthquake.

\section{METHODS}

\section{Participants}

The data used in this cross-sectional study were obtained from the Fukushima Health Management Survey. The target population was 210189 evacuees aged $>0$ years at the time of the earthquake, who were registered officially in fiscal year 2011 from evacuation zones including Hirono Town, Naraha Town, Tomioka Town, Kawauchi Village, Okuma Town, Futaba Town, Namie Town,
Katsurao Village, Minamisoma City, Tamura City, Yamakiya district of Kawamata Town and Iitate Village. Of these, 180604 evacuees were over 15 years old. In total, 73433 people aged 15 years and older responded to and were included in the Fukushima Health Management Survey of survivors of the Great East Japan Earthquake from the evacuation zones (response rate: $40.7 \%)^{4}$

We excluded participants with missing information for variables related to psychological distress $(n=7567)$ or with more than three missing pieces of information for questions about dietary intake $(\mathrm{n}=2819)$, leaving 63047 participants (27 901 men, 35146 women) for inclusion in the present analysis.

The study protocol was approved by the Ethics Review Committee of Fukushima Medical University (number 1316).$^{5}$

\section{Assessment of mental health status}

To assess the mental health status of participants, we used the Kessler 6 (K6) scale ${ }^{11}$ and the Post-traumatic Stress Disorder (PTSD) Checklist-Stressor-Specific Version (PCL-S). ${ }^{12}$ Of the respondents to the questionnaire, $88.6 \%$ responded directly, while $9.3 \%$ responded by proxy and $2.1 \%$ of questionnaires were missing this information.

The K6 scale was used to measure non-specific mental health distress. This scale asks participants if they have experienced any of the following six symptoms during the past 30 days: 'feeling so sad that nothing could cheer you up', 'feeling nervous', 'hopeless', 'restless or fidgety', 'feeling everything was an effort' and 'feeling worthless'. Each question was scored on a five-point Likert-type scale from 0 to 4 , with higher scores meaning worse mental health status. Scores thus ranged from 0 to 24. The Japanese version of the $\mathrm{K} 6$ has been validated. ${ }^{13}$ We defined non-specific mental health distress as corresponding to a K6 score of $\geq 13$. ${ }^{11}$

The PCL-S was used to measure traumatic symptoms caused by the experience of the Great East Japan Earthquake and the following accidents. The PCL-S is a 17-item self-report measure designed to detect PTSD, where each item is scored from 1 to 5 , corresponding to 'not at all', 'a little bit', 'moderately', 'quite a bit' or 'extremely', respectively. We classified participants as having probable PTSD if their total PCL-S score was $\geq 44^{12}$

\section{Assessment of dietary intake}

Participants' food intake was assessed by using a food frequency questionnaire (FFQ). We modified a reference FFQ used in studies conducted by the Radiation Effects Research Foundation ${ }^{15}$ and selected 19 items. The current frequency of consumption for these items was then assessed.

The 19 items were divided into 10 groups: rice and bread, fish (sashimi, cooked/boiled/fried fish), meat (chicken, beef, pork, ham, sausages), vegetables (green 
vegetables, red and orange vegetables, light-coloured vegetables), vegetable juice, fruits, fruit juice, soya bean products (natto fermented soya beans, miso soup, tofu dishes, boiled bean dishes, soya milk), milk and yogurt, and lactobacillus drinks. Food intake frequency was assessed in terms of the following six categories: everyday, 5-6 times/week, 3-4 times/week, 1-2 times/week, less than once/week and never.

To calculate daily food intake, the daily midpoint for each frequency category was used. For instance, '3-4 times/week' was evaluated as 0.5 times/day. The frequency of consumption of each food was categorised by quartile, and the frequencies in the 25th centile (Q1) were lowest. Daily food consumption in the 25th centile or lower was defined as 'low frequency' for the corresponding food group.

\section{Disaster-related and other variables}

Participants' basic characteristics and disaster-related factors were obtained through the self-report questionnaire. Smoking status was classified into three categories: current, former and never. Drinking status was similarly classified: once or more/month, previous drinker or less than once/month. Perceived health condition was selfreported as one of five categories: very good, good, normal, poor and very poor. History of mental illness and history of other illness were both answered by a dichotomised variable (yes, no). Leisure-time physical activity was classified into four categories: almost every day, 2-4 times/week, once/week and almost never. Sleep habits were classified into four categories: satisfied, slightly dissatisfied, quite dissatisfied and very dissatisfied/have not slept at all. Educational attainment was classified into four groups: elementary or junior high school, high school, vocational or junior college and university or graduate school. Age was categorised into five groups: $15-44,45-54,55-64,65-74$ and 75 years and older. Having experienced the Great East Japan Earthquake was answered with a 'yes' or 'no'. There were six options for living arrangements at the time of the survey, which were classified into two groups for analysis: 'evacuation shelter or temporary housing' and 'rental housing or relative's home, own home, or other'. Changes in work situation since the disaster, loss of employment, decrease in income and death of someone close were answered with a 'yes' or 'no'.

\section{Statistical analysis}

Participants' characteristics were compared using $\chi^{2}$ tests between participants with habitual intake of a given food group and participants without it, for each food group.

Logistic regression analysis was used to investigate the association between psychological distress and low frequency of consumption of certain foods, in terms of estimated ORs and 95\% CIs. Model 1 was adjusted for age and sex. Model 2 consisted of the parameters of model 1 plus the variables of smoking and drinking statuses, perceived health condition, history of mental illness, history of other illness, leisure-time physical activity, sleeping habits, educational attainment, experiences of specific events during the disaster, living arrangements, changes in working situation, loss of employment, decreased income and death of someone close.

All statistical analyses were conducted with SPSS Statistics for Windows, V.23.0 software (IBM, Armonk, New York, New York, USA). All tests were two-sided: $\mathrm{p}<0.05$ was considered statistically significant.

\section{RESULTS}

Of the 63047 participants included in our analysis, $14.7 \%$ had a $\mathrm{K} 6$ score of $\geq 13$ and $21.2 \%$ had a PCL-S score of $\geq 44$. Above-threshold scores were more common in women than in men $(12.1 \%$ vs $16.7 \%$, and $17.5 \%$ vs $23.6 \%$, respectively).

Table 1 shows the demographic, lifestyle-related and disaster-related characteristics of participants stratified according to the frequency of food consumption status for different food groups. Compared with older participants, younger participants tended to have a low frequency of consumption of rice and bread, fish, vegetables (non-juice), fruit (non-juice), soya bean products and milk. Assuming the high-consumption versus low-consumption cut-offs described in the column headings in table 1 , for most food groups participants with the lower frequency of the pair were more likely to: drink once or more per month, be a current smoker, not perform any physical activities in their leisure time and have suffered decreased income.

The frequency of food consumption and percentage of participants with a low frequency of food consumption are shown in table 2 . In both sexes, a low frequency of consumption was more common in participants with $\mathrm{K} 6 \geq 13$ than in participants with $\mathrm{K} 6<13$ for rice and bread, fish, meat, vegetables (non-juice), fruits (nonjuice), soya bean products, milk and yogurt or lactobacillus drinks. Moreover, a low frequency of food consumption was more common in participants with PCL $\geq 44$ than in participants with $\mathrm{PCL}<44$ for rice and bread, meat and milk. Conversely, a low frequency of food consumption was more common in participants with PCL $<44$ than in participants with PCL $\geq 44$ for vegetable juice and fruit juice (all $\mathrm{p}<0.05$ ).

In age-adjusted logistic models, non-specific mental health distress was positively associated with a low frequency of food consumption for rice and bread, fish, meat, vegetables (non-juice), fruits (non-juice), soya bean products, milk and yogurt or lactobacillus drinks (model 1) (table 3). In the multivariable adjusted model, these positive associations between non-specific mental health distress and low frequency of food consumption were also confirmed for rice and bread, fish, meat, vegetables (non-juice), fruits (non-juice), soya bean products, milk and yogurt and/or lactobacillus drinks; 1.16 (1.08 to 1.25$), 1.22$ (1.12 to 1.32 ), 1.09 (1.02 to 1.17 ), 1.12 (1.04 to 1.20$), 1.13$ (1.05 to 1.21 ), 1.08 
Table 1 Participant characteristics according to frequency of food consumption status (2011 data)

\begin{tabular}{|c|c|c|c|c|c|c|c|c|c|c|c|c|c|c|c|c|}
\hline & \multicolumn{16}{|c|}{ Number (\%) } \\
\hline & \multirow[b]{2}{*}{ Total } & \multicolumn{3}{|c|}{ Rice and bread } & \multicolumn{3}{|l|}{ Fish } & \multicolumn{3}{|l|}{ Meat } & \multicolumn{3}{|c|}{ Vegetables (non-juice) } & \multicolumn{3}{|c|}{ Vegetable juice } \\
\hline & & Daily & $\begin{array}{l}5-6 \\
\text { times/ } \\
\text { week or } \\
\text { less }\end{array}$ & $\begin{array}{l}\text { p } \\
\text { value }\end{array}$ & $\begin{array}{l}3-4 \\
\text { times/ } \\
\text { week or } \\
\text { more }\end{array}$ & $\begin{array}{l}1-2 \\
\text { times/ } \\
\text { week or } \\
\text { less }\end{array}$ & $\begin{array}{l}\text { p } \\
\text { value }^{*}\end{array}$ & $\begin{array}{l}3-4 \\
\text { times/ } \\
\text { week or } \\
\text { more }\end{array}$ & $\begin{array}{l}1-2 \\
\text { times/ } \\
\text { week or } \\
\text { less }\end{array}$ & $\begin{array}{l}\text { p } \\
\text { value }^{*}\end{array}$ & Daily & $\begin{array}{l}\text { 5-6 } \\
\text { times/ } \\
\text { week or } \\
\text { less }\end{array}$ & $\begin{array}{l}p \\
\text { value }^{*}\end{array}$ & $\begin{array}{l}\text { Less than } \\
\text { once/ } \\
\text { week or } \\
\text { more }\end{array}$ & Never & $\begin{array}{l}\text { p } \\
\text { value }\end{array}$ \\
\hline \multicolumn{17}{|l|}{ Sex $(n=63047)$} \\
\hline Men & 27901 & $\begin{array}{l}26375 \\
(44.2)\end{array}$ & $\begin{array}{l}1526 \\
(45.3)\end{array}$ & 0.10 & $\begin{array}{l}14597 \\
(43.9)\end{array}$ & $\begin{array}{l}13304 \\
(44.6)\end{array}$ & 0.045 & $\begin{array}{l}16454 \\
(42.7)\end{array}$ & $\begin{array}{l}11447 \\
(46.8)\end{array}$ & $<0.001$ & $\begin{array}{l}16620 \\
(39.6)\end{array}$ & $\begin{array}{l}11447 \\
(46.8)\end{array}$ & $<0.001$ & $\begin{array}{l}14754 \\
(44.3)\end{array}$ & $\begin{array}{l}13151 \\
(44.1)\end{array}$ & 0.31 \\
\hline \multicolumn{17}{|l|}{ Age (years) $(n=63047)$} \\
\hline $15-44$ & 20384 & $\begin{array}{l}18699 \\
(31.3)\end{array}$ & $\begin{array}{l}1685 \\
(50.1)\end{array}$ & $<0.001$ & $\begin{array}{l}7087 \\
(21.3)\end{array}$ & $\begin{array}{l}13297 \\
(44.6)\end{array}$ & $<0.001$ & $\begin{array}{l}15367 \\
(39.8)\end{array}$ & $\begin{array}{l}5017 \\
(20.5)\end{array}$ & $<0.001$ & $\begin{array}{l}11560 \\
(27.6)\end{array}$ & $\begin{array}{l}8824 \\
(41.8)\end{array}$ & $<0.001$ & $\begin{array}{l}10795 \\
(32.5)\end{array}$ & $\begin{array}{l}9589 \\
(32.2)\end{array}$ & $<0.001$ \\
\hline $45-54$ & 8923 & $\begin{array}{l}8410 \\
(14.1)\end{array}$ & $\begin{array}{l}513 \\
(15.2)\end{array}$ & & $\begin{array}{l}4130 \\
(12.4)\end{array}$ & $\begin{array}{l}4793 \\
(16.1)\end{array}$ & & $\begin{array}{l}5585 \\
(14.5)\end{array}$ & $\begin{array}{l}3338 \\
(13.6)\end{array}$ & & $\begin{array}{l}5217 \\
(12.4)\end{array}$ & $\begin{array}{l}3706 \\
(17.6)\end{array}$ & & $\begin{array}{l}4401 \\
(13.2)\end{array}$ & $\begin{array}{l}4522 \\
(15.2)\end{array}$ & \\
\hline $55-64$ & 14504 & $\begin{array}{l}13871 \\
(23.2)\end{array}$ & $\begin{array}{l}633 \\
(18.8)\end{array}$ & & $\begin{array}{l}8781 \\
(26.4)\end{array}$ & $\begin{array}{l}5723 \\
(19.2)\end{array}$ & & $\begin{array}{l}7704 \\
(20.0)\end{array}$ & $\begin{array}{l}6800 \\
(27.8)\end{array}$ & & $\begin{array}{l}9819 \\
(23.4)\end{array}$ & $\begin{array}{l}4685 \\
(22.2)\end{array}$ & & $\begin{array}{l}7564 \\
(22.7)\end{array}$ & $\begin{array}{l}6940 \\
(23.3)\end{array}$ & \\
\hline $65-74$ & 10378 & $\begin{array}{l}10076 \\
(16.9)\end{array}$ & $302(9.0)$ & & $\begin{array}{l}7246 \\
(21.8)\end{array}$ & $\begin{array}{l}3132 \\
(10.5)\end{array}$ & & $\begin{array}{l}5322 \\
(13.8)\end{array}$ & $\begin{array}{l}5056 \\
(20.7)\end{array}$ & & $\begin{array}{l}8177 \\
(19.5)\end{array}$ & $\begin{array}{l}2201 \\
(10.4)\end{array}$ & & $\begin{array}{l}5808 \\
(17.5)\end{array}$ & $\begin{array}{l}4570 \\
(15.3)\end{array}$ & \\
\hline$\geqq 75$ & 8858 & $\begin{array}{l}8626 \\
(14.5)\end{array}$ & $232(6.9)$ & & $\begin{array}{l}5980 \\
(18.0)\end{array}$ & $\begin{array}{l}2878 \\
(9.7)\end{array}$ & & $\begin{array}{l}4596 \\
(11.9)\end{array}$ & $\begin{array}{l}4262 \\
(17.4)\end{array}$ & & $\begin{array}{l}7185 \\
(17.1)\end{array}$ & $\begin{array}{l}1673 \\
(7.9)\end{array}$ & & $\begin{array}{l}4691 \\
(14.1)\end{array}$ & $\begin{array}{l}4157 \\
(14.0)\end{array}$ & \\
\hline \multicolumn{17}{|l|}{ Drinking status ( $n=62343$ ) } \\
\hline Once or more per month & 27734 & $\begin{array}{l}25906 \\
(43.9)\end{array}$ & $\begin{array}{l}1828 \\
(54.8)\end{array}$ & $<0.001$ & $\begin{array}{l}14744 \\
(45.0)\end{array}$ & $\begin{array}{l}12990 \\
(43.9)\end{array}$ & $<0.001$ & $\begin{array}{l}16915 \\
(44.3)\end{array}$ & $\begin{array}{l}10819 \\
(44.8)\end{array}$ & $<0.001$ & $\begin{array}{l}17504 \\
(42.2)\end{array}$ & $\begin{array}{l}10230 \\
(48.9)\end{array}$ & $<0.001$ & $\begin{array}{l}14332 \\
(443.6)\end{array}$ & $\begin{array}{l}13402 \\
(45.4)\end{array}$ & $<0.001$ \\
\hline \multicolumn{17}{|l|}{ Smoking status ( $n=61933$ ) } \\
\hline Current & 13402 & $\begin{array}{l}12333 \\
(21.0)\end{array}$ & $\begin{array}{l}1069 \\
(32.3)\end{array}$ & $<0.001$ & $\begin{array}{l}5895 \\
(18.1)\end{array}$ & $\begin{array}{l}7507 \\
(25.5)\end{array}$ & $<0.001$ & $\begin{array}{l}8430 \\
(22.2)\end{array}$ & $\begin{array}{l}4972 \\
(20.7)\end{array}$ & $<0.001$ & $\begin{array}{l}7106 \\
(17.3)\end{array}$ & $\begin{array}{l}6296 \\
(30.3)\end{array}$ & $<0.001$ & $\begin{array}{l}6428 \\
(19.7)\end{array}$ & $\begin{array}{l}6974 \\
(23.8)\end{array}$ & $<0.001$ \\
\hline \multicolumn{17}{|l|}{ Health condition ( $n=62009)$} \\
\hline Poor/very poor & 11547 & $\begin{array}{l}10716 \\
(18.2)\end{array}$ & $\begin{array}{l}831 \\
(25.1)\end{array}$ & $<0.001$ & $\begin{array}{l}6324 \\
(19.4)\end{array}$ & $\begin{array}{l}5223 \\
(17.8)\end{array}$ & $<0.001$ & $\begin{array}{l}6404 \\
(16.9)\end{array}$ & $\begin{array}{l}5143 \\
(21.4)\end{array}$ & $<0.001$ & $\begin{array}{l}7614 \\
(18.5)\end{array}$ & $\begin{array}{l}3933 \\
(18.9)\end{array}$ & $<0.001$ & $\begin{array}{l}6223 \\
(19.0)\end{array}$ & $\begin{array}{l}5324 \\
(18.2)\end{array}$ & 0.01 \\
\hline \multicolumn{17}{|c|}{ History of mental illness ( $n=61239)$} \\
\hline Yes & 3406 & $\begin{array}{l}3129 \\
(5.4)\end{array}$ & $277(8.6)$ & $<0.001$ & $\begin{array}{l}1767 \\
(5.5)\end{array}$ & $\begin{array}{l}1639 \\
(5.7)\end{array}$ & 0.16 & $\begin{array}{l}1929 \\
(5.1)\end{array}$ & $\begin{array}{l}1477 \\
(6.2)\end{array}$ & $<0.001$ & $\begin{array}{l}2171 \\
(5.3)\end{array}$ & $\begin{array}{l}1235 \\
(6.0)\end{array}$ & $<0.001$ & $1852(5.7)$ & $\begin{array}{l}1554 \\
(5.4)\end{array}$ & 0.02 \\
\hline \multicolumn{17}{|c|}{ History of other illness $(n=63047)$} \\
\hline Yes & 62912 & $\begin{array}{l}59566 \\
(99.8)\end{array}$ & $\begin{array}{l}3346 \\
(99.4)\end{array}$ & $<0.001$ & $\begin{array}{l}33156 \\
(99.8)\end{array}$ & $\begin{array}{l}29756 \\
(99.8)\end{array}$ & 0.32 & $\begin{array}{l}38496 \\
(99.8)\end{array}$ & $\begin{array}{l}24416 \\
(99.8)\end{array}$ & 0.23 & $\begin{array}{l}41882 \\
(99.8)\end{array}$ & $\begin{array}{l}21030 \\
(99.7)\end{array}$ & 0.01 & $\begin{array}{l}33178 \\
(99.8)\end{array}$ & $\begin{array}{l}29734 \\
(99.8)\end{array}$ & 0.06 \\
\hline \multicolumn{17}{|c|}{ Leisure-time physical activity ( $n=62150)$} \\
\hline Almost never & 33060 & $\begin{array}{l}31082 \\
(52.8)\end{array}$ & $\begin{array}{l}1978 \\
(59.5)\end{array}$ & $<0.001$ & $\begin{array}{l}15152 \\
(46.4)\end{array}$ & $\begin{array}{l}17908 \\
(60.6)\end{array}$ & $<0.001$ & $\begin{array}{l}20875 \\
(54.8)\end{array}$ & $\begin{array}{l}12185 \\
(50.6)\end{array}$ & $<0.001$ & $\begin{array}{l}19812 \\
(48.0)\end{array}$ & $\begin{array}{l}13248 \\
(63.4)\end{array}$ & $<0.001$ & $\begin{array}{l}16010 \\
(48.9)\end{array}$ & $\begin{array}{l}17050 \\
(58.0)\end{array}$ & $<0.001$ \\
\hline \multicolumn{17}{|c|}{ Sleeping habits $(n=52474)$} \\
\hline $\begin{array}{l}\text { Very dissatisfied or have } \\
\text { not slept at all }\end{array}$ & 2254 & $\begin{array}{l}2000 \\
(4.0)\end{array}$ & $254(8.9)$ & $<0.001$ & $\begin{array}{l}1116 \\
(4.1)\end{array}$ & $\begin{array}{l}1138 \\
(4.5)\end{array}$ & $<0.001$ & $\begin{array}{l}1255 \\
(3.9)\end{array}$ & $999(5.0)$ & $<0.001$ & $\begin{array}{l}1370 \\
(4.0)\end{array}$ & $884(5.0)$ & $<0.001$ & $1222(4.4)$ & $\begin{array}{l}1032 \\
(4.1)\end{array}$ & $<0.001$ \\
\hline \multicolumn{17}{|c|}{ Educational attainment $(\mathrm{n}=60998)$} \\
\hline $\begin{array}{l}\text { University/graduate } \\
\text { school }\end{array}$ & 5457 & $\begin{array}{l}5096 \\
(8.8)\end{array}$ & $\begin{array}{l}361 \\
(11.2)\end{array}$ & $<0.001$ & $\begin{array}{l}2596 \\
(8.1)\end{array}$ & $\begin{array}{l}2861 \\
(9.9)\end{array}$ & $<0.001$ & $\begin{array}{l}3697 \\
(9.9)\end{array}$ & $\begin{array}{l}1760 \\
(7.5)\end{array}$ & $<0.001$ & $\begin{array}{l}3749 \\
(9.2)\end{array}$ & $\begin{array}{l}1708 \\
(8.3)\end{array}$ & $<0.001$ & $3030(9.4)$ & $\begin{array}{l}2427 \\
(8.4)\end{array}$ & $<0.001$ \\
\hline
\end{tabular}




\begin{tabular}{|c|c|c|c|c|c|c|c|c|c|c|c|c|c|c|c|c|c|c|c|c|}
\hline & \multicolumn{20}{|c|}{ Number (\%) } \\
\hline & \multirow[b]{2}{*}{ Total } & \multicolumn{4}{|c|}{ Rice and bread } & \multicolumn{4}{|l|}{ Fish } & \multicolumn{5}{|c|}{ Meat } & \multicolumn{3}{|c|}{ Vegetables (non-juice) } & \multicolumn{3}{|c|}{ Vegetable juice } \\
\hline & & Daily & \multicolumn{2}{|c|}{$\begin{array}{l}5-6 \\
\text { times/ } \\
\text { week or } \\
\text { less } \\
\end{array}$} & $\begin{array}{l}p \\
\text { value }\end{array}$ & $\begin{array}{l}3-4 \\
\text { times/ } \\
\text { week or } \\
\text { more } \\
\end{array}$ & \multicolumn{2}{|c|}{$\begin{array}{l}1-2 \\
\text { times/ } \\
\text { week or } \\
\text { less } \\
\end{array}$} & $\begin{array}{l}\mathrm{p} \\
\text { value* }\end{array}$ & \multicolumn{2}{|c|}{$\begin{array}{l}3-4 \\
\text { times/ } \\
\text { week or } \\
\text { more }\end{array}$} & \multicolumn{2}{|c|}{$\begin{array}{l}1-2 \\
\text { times/ } \\
\text { week or } \\
\text { less } \\
\end{array}$} & \multirow[t]{2}{*}{$\begin{array}{l}\text { p } \\
\text { value* }\end{array}$} & Daily & \multirow[t]{2}{*}{$\begin{array}{l}5-6 \\
\text { times/ } \\
\text { week or } \\
\text { less } \\
\end{array}$} & \multirow[t]{2}{*}{$\begin{array}{l}\text { p } \\
\text { value* }\end{array}$} & $\begin{array}{l}\text { Less than } \\
\text { once/ } \\
\text { week or } \\
\text { more }\end{array}$ & Never & $\begin{array}{l}p \\
\text { value }\end{array}$ \\
\hline \multirow{2}{*}{\multicolumn{21}{|c|}{$\begin{array}{l}\text { Experiences of the Great East Japan Earthquake } \\
\text { Earthquake }(\mathrm{n}=63 \text { 047) }\end{array}$}} \\
\hline & & & & & & & & & & & & & & & & & & & & \\
\hline Yes & 61014 & $\begin{array}{l}57769 \\
(96.8)\end{array}$ & $\begin{array}{l}3245 \\
(96.4)\end{array}$ & & 0.14 & $\begin{array}{l}32055 \\
(96.5)\end{array}$ & $\begin{array}{l}28959 \\
(97.1)\end{array}$ & & $<0.001$ & $\begin{array}{l}3742 \\
(97.0\end{array}$ & & $\begin{array}{l}235 \\
(96.4\end{array}$ & & $<0.001$ & $\begin{array}{l}40596 \\
(96.8)\end{array}$ & $\begin{array}{l}20418 \\
(96.8)\end{array}$ & 0.68 & $\begin{array}{l}32143 \\
(96.6)\end{array}$ & $\begin{array}{l}28871 \\
(96.9)\end{array}$ & 0.03 \\
\hline \multicolumn{21}{|l|}{ Tsunami (n=63 047) } \\
\hline Yes & 12435 & $\begin{array}{l}11690 \\
(19.6)\end{array}$ & $\begin{array}{l}745 \\
(22.1)\end{array}$ & & 0.001 & $\begin{array}{l}6767 \\
(20.4)\end{array}$ & $\begin{array}{l}5668 \\
(19.0)\end{array}$ & & $=0.001$ & $\begin{array}{l}7350 \\
(19.1\end{array}$ & & $\begin{array}{l}5085 \\
(20.8\end{array}$ & & $<0.001$ & $\begin{array}{l}8074 \\
(19.2)\end{array}$ & $\begin{array}{l}4361 \\
(20.7)\end{array}$ & $<0.001$ & $\begin{array}{ll}1 & 6868 \\
(20.7)\end{array}$ & $\begin{array}{l}5567 \\
(18.7)\end{array}$ & $<0.001$ \\
\hline Nuclear reactor acciden & $=63047)$ & & & & & & & & & & & & & & & & & & & \\
\hline Yes & 32846 & $\begin{array}{l}30880 \\
(51.7)\end{array}$ & $\begin{array}{l}1966 \\
(58.4)\end{array}$ & & 0.001 & $\begin{array}{l}17967 \\
(54.1)\end{array}$ & $\begin{array}{l}14879 \\
(49.9)\end{array}$ & & 0.001 & $\begin{array}{l}1966 \\
(51.0\end{array}$ & & $\begin{array}{l}1318 \\
(53.9\end{array}$ & & $<0.001$ & $\begin{array}{l}22108 \\
(52.7)\end{array}$ & $\begin{array}{l}10738 \\
(50.9)\end{array}$ & $<0.001$ & $\begin{array}{l}17655 \\
(53.1)\end{array}$ & $\begin{array}{l}15191 \\
(51.0)\end{array}$ & $<0.001$ \\
\hline None $(n=63047)$ & & & & & & & & & & & & & & & & & & & & \\
\hline Yes & 696 & $\begin{array}{l}663 \\
(1.1)\end{array}$ & $33(1$. & & 0.27 & $378(1.1)$ & $318(1$. & & 0.21 & 402( & (1.0) & 294 & (1.2) & 0.03 & $\begin{array}{l}480 \\
(1.1)\end{array}$ & $216(1.0)$ & 0.18 & $396(1.2)$ & $\begin{array}{l}300 \\
(1.0)\end{array}$ & 0.02 \\
\hline Living arrangements $(\mathrm{n}=$ & 047) & & & & & & & & & & & & & & & & & & & \\
\hline $\begin{array}{l}\text { Evacuation shelter or } \\
\text { temporary housing }\end{array}$ & 6599 & $\begin{array}{l}6206 \\
(10.4)\end{array}$ & $\begin{array}{l}393 \\
(11.7)\end{array}$ & & 0.01 & $\begin{array}{l}3598 \\
(10.8)\end{array}$ & $\begin{array}{l}3001 \\
(10.1)\end{array}$ & & 0.001 & $\begin{array}{l}3659 \\
(9.5)\end{array}$ & & $\begin{array}{l}2940 \\
(12.0\end{array}$ & & $<0.001$ & $\begin{array}{l}4175 \\
(10.0)\end{array}$ & $\begin{array}{l}2424 \\
(11.5)\end{array}$ & $<0.001$ & $\begin{array}{l}3662 \\
(11.0)\end{array}$ & $\begin{array}{l}2937 \\
(9.9)\end{array}$ & $<0.001$ \\
\hline Changes in work situatic & $n=60077$ & & & & & & & & & & & & & & & & & & & \\
\hline Yes & 32118 & $\begin{array}{l}30074 \\
(52.9)\end{array}$ & $\begin{array}{l}2044 \\
(63.0)\end{array}$ & & 0.001 & $\begin{array}{l}16160 \\
(51.5)\end{array}$ & $\begin{array}{l}15958 \\
(55.7)\end{array}$ & & 0.001 & $\begin{array}{l}1997 \\
(54.2\end{array}$ & & $\begin{array}{l}121 \\
(52.2\end{array}$ & & $<0.001$ & $\begin{array}{l}20634 \\
(51.9)\end{array}$ & $\begin{array}{l}11484 \\
(56.5)\end{array}$ & $<0.001$ & $\begin{array}{l}1 \quad 16981 \\
(53.7)\end{array}$ & $\begin{array}{l}15137 \\
(53.2)\end{array}$ & 0.08 \\
\hline Loss of employment $(n=$ & 047) & & & & & & & & & & & & & & & & & & & \\
\hline Yes & 12702 & $\begin{array}{l}11812 \\
(19.8)\end{array}$ & $\begin{array}{l}890 \\
(26.4)\end{array}$ & & 0.001 & $\begin{array}{l}6408 \\
(19.3)\end{array}$ & $\begin{array}{l}6294 \\
(21.1)\end{array}$ & & 0.001 & $\begin{array}{l}7887 \\
(20.4\end{array}$ & & $\begin{array}{l}4815 \\
(19.7\end{array}$ & & $<0.01$ & $\begin{array}{l}8285 \\
(19.7)\end{array}$ & $\begin{array}{l}4417 \\
(20.9)\end{array}$ & $<0.001$ & $\begin{array}{ll}1 & 6569 \\
(19.8)\end{array}$ & $\begin{array}{l}6133 \\
(20.6)\end{array}$ & 0.01 \\
\hline Decreased income $(n=6$ & & & & & & & & & & & & & & & & & & & & \\
\hline & 11513 & $\begin{array}{l}10829 \\
(18.1)\end{array}$ & $\begin{array}{l}684 \\
(20.3)\end{array}$ & & 0.001 & $\begin{array}{l}5789 \\
(17.4)\end{array}$ & $\begin{array}{l}5724 \\
(19.2)\end{array}$ & & 0.001 & $\begin{array}{l}7178 \\
(18.6\end{array}$ & & $\begin{array}{l}4335 \\
(17.7\end{array}$ & & $<0.001$ & $\begin{array}{l}7235 \\
(17.2)\end{array}$ & $\begin{array}{l}4278 \\
(20.3)\end{array}$ & $<0.001$ & $\begin{array}{ll}1 & 6063 \\
(18.2)\end{array}$ & $\begin{array}{l}5450 \\
(18.3)\end{array}$ & 0.42 \\
\hline Death of someone close & $=62081)$ & & & & & & & & & & & & & & & & & & & \\
\hline Yes & 12006 & $\begin{array}{l}11287 \\
(19.2)\end{array}$ & $\begin{array}{l}719 \\
(21.8)\end{array}$ & & 0.001 & $\begin{array}{l}6602 \\
(20.2)\end{array}$ & $\begin{array}{l}5404 \\
(18.4)\end{array}$ & & 0.001 & $\begin{array}{l}7264 \\
(19.1\end{array}$ & & $\begin{array}{l}4742 \\
(19.7\end{array}$ & & 0.04 & $\begin{array}{l}8092 \\
(19.6)\end{array}$ & $\begin{array}{l}3914 \\
(18.8)\end{array}$ & 0.03 & $\begin{array}{l}6574 \\
(20.1)\end{array}$ & $\begin{array}{l}5432 \\
(18.5)\end{array}$ & $<0.001$ \\
\hline & Fruits $(r$ & non-juice & & & & it juice & & & Soy & ya bea & an proc & ducts & & Milk & & & & $\begin{array}{l}\text { Yogurt and } \\
\text { drinks }\end{array}$ & lactobacillus & \\
\hline & $\begin{array}{l}1-2 \\
\text { times/ } \\
\text { week or } \\
\text { more }\end{array}$ & $\begin{array}{l}\text { Less } \\
\text { once } \\
\text { or } n e\end{array}$ & $\begin{array}{l}\text { than } \\
\text { /week } \\
\text { ever }\end{array}$ & $\begin{array}{l}\text { p } \\
\text { value* }\end{array}$ & $\begin{array}{r}\text { Less } \\
\text { onc } \\
\text { * or } \mathrm{m} \\
\end{array}$ & $\begin{array}{l}\text { ss than } \\
\text { ce/week } \\
\text { more }\end{array}$ & Never & $\begin{array}{l}\mathbf{p} \\
\text { value* }\end{array}$ & * Dail & & $\begin{array}{l}5-6 \\
\text { times } \\
\text { week } \\
\text { less } \\
\end{array}$ & & $\begin{array}{l}p \\
\text { value* }\end{array}$ & $\begin{array}{l}1-2 \\
\text { times } \\
\text { week } \\
\text { more } \\
\end{array}$ & sl & $\begin{array}{l}\text { Less than } \\
\text { once/week } \\
\text { or never }\end{array}$ & $\begin{array}{ll} & 1 \\
& t \\
p & v \\
\text { value }^{*} & n \\
\end{array}$ & $\begin{array}{l}1-2 \\
\text { times/ } \\
\text { week or } \\
\text { more }\end{array}$ & $\begin{array}{l}\text { Less than } \\
\text { once/week } \\
\text { or never }\end{array}$ & $\begin{array}{l}\text { p } \\
\text { value }\end{array}$ \\
\hline Sex $(n=63047)$ & & & & & & & & & & & & & & & & & & & & \\
\hline Men & $\begin{array}{l}17332 \\
(38.9)\end{array}$ & $\begin{array}{l}1056 \\
(57.0\end{array}$ & & $<0.001$ & $\begin{array}{ll}1 & 163 \\
& (44.3\end{array}$ & 397 & $\begin{array}{l}11504 \\
(44.2)\end{array}$ & 0.48 & $\begin{array}{l}215 \\
(43 . \varepsilon\end{array}$ & $\begin{array}{l}517 \\
.8)\end{array}$ & $\begin{array}{l}6384 \\
(45.7)\end{array}$ & & $<0.001$ & $\begin{array}{l}1 \quad 1935 \\
(42.8)\end{array}$ & & $\begin{array}{l}8545 \\
(47.8)\end{array}$ & $<0.001$ & $\begin{array}{l}21089 \\
(40.1)\end{array}$ & $\begin{array}{l}6812 \\
(65.2)\end{array}$ & $<0.001$ \\
\hline Age (years) $(n=63$ 047) & & & & & & & & & & & & & & & & & & & & \\
\hline $15-44$ & $\begin{array}{l}11061 \\
(24.8)\end{array}$ & $\begin{array}{l}9323 \\
(50.3\end{array}$ & & $<0.001$ & $\begin{array}{ll}1 & 131 \\
& (35.4\end{array}$ & $\begin{array}{l}122 \\
4)\end{array}$ & $\begin{array}{l}7262 \\
(27.9)\end{array}$ & $<0.001$ & $\begin{array}{ll}1 & 132 \\
& \\
& (27 .\end{array}$ & $\begin{array}{l}264 \\
.0)\end{array}$ & $\begin{array}{l}7120 \\
(51.0)\end{array}$ & & $<0.001$ & $\begin{array}{ll}1 & 1443 \\
& (32.0)\end{array}$ & & $\begin{array}{l}5948 \\
(33.3)\end{array}$ & $<0.001$ & $\begin{array}{l}17075 \\
(32.5)\end{array}$ & $\begin{array}{l}3309 \\
(31.7)\end{array}$ & $<0.01$ \\
\hline
\end{tabular}




\begin{tabular}{|c|c|c|c|c|c|c|c|c|c|c|c|c|c|c|c|}
\hline & \multicolumn{3}{|c|}{ Fruits (non-juice) } & \multicolumn{3}{|l|}{ Fruit juice } & \multicolumn{3}{|c|}{ Soya bean products } & \multicolumn{3}{|l|}{ Milk } & \multicolumn{3}{|c|}{$\begin{array}{l}\text { Yogurt and lactobacillus } \\
\text { drinks }\end{array}$} \\
\hline & $\begin{array}{l}1-2 \\
\text { times/ } \\
\text { week or } \\
\text { more } \\
\end{array}$ & $\begin{array}{l}\text { Less than } \\
\text { once/week } \\
\text { or never }\end{array}$ & $\begin{array}{l}\text { p } \\
\text { value* }^{*}\end{array}$ & $\begin{array}{l}\text { Less than } \\
\text { once/week } \\
\text { or more }\end{array}$ & Never & $\begin{array}{l}\text { p } \\
\text { value* }^{*}\end{array}$ & Daily & $\begin{array}{l}5-6 \\
\text { times/ } \\
\text { week or } \\
\text { less } \\
\end{array}$ & $\begin{array}{l}\text { p } \\
\text { value* }^{*}\end{array}$ & $\begin{array}{l}1-2 \\
\text { times/ } \\
\text { week or } \\
\text { more } \\
\end{array}$ & $\begin{array}{l}\text { Less than } \\
\text { once/week } \\
\text { or never }\end{array}$ & $\begin{array}{l}\mathrm{p} \\
\text { value* }^{*}\end{array}$ & $\begin{array}{l}1-2 \\
\text { times/ } \\
\text { week or } \\
\text { more } \\
\end{array}$ & $\begin{array}{l}\text { Less than } \\
\text { once/week } \\
\text { or never }\end{array}$ & $\begin{array}{l}\text { p } \\
\text { value* }\end{array}$ \\
\hline $45-54$ & $\begin{array}{l}5754 \\
(12.9)\end{array}$ & $\begin{array}{l}3169 \\
(17.1)\end{array}$ & & $\begin{array}{l}4907 \\
(13.2)\end{array}$ & $\begin{array}{l}4016 \\
(15.4)\end{array}$ & & $\begin{array}{l}6577 \\
(13.4)\end{array}$ & $\begin{array}{l}2346 \\
(16.8)\end{array}$ & & $\begin{array}{l}6188 \\
(13.7)\end{array}$ & $\begin{array}{l}2735 \\
(15.3)\end{array}$ & & $\begin{array}{l}7363 \\
(14.0)\end{array}$ & $\begin{array}{l}1560 \\
(14.9)\end{array}$ & \\
\hline $55-64$ & $\begin{array}{l}11125 \\
(25.0)\end{array}$ & $\begin{array}{l}3379 \\
(18.2)\end{array}$ & & $\begin{array}{l}7915 \\
(21.4)\end{array}$ & $\begin{array}{l}6589 \\
(25.3)\end{array}$ & & $\begin{array}{l}12007 \\
(24.5)\end{array}$ & $\begin{array}{l}2497 \\
(17.9)\end{array}$ & & $\begin{array}{l}10409 \\
(23.0)\end{array}$ & $\begin{array}{l}4095 \\
(22.9)\end{array}$ & & $\begin{array}{l}12056 \\
(22.9)\end{array}$ & $\begin{array}{l}2448 \\
(23.4)\end{array}$ & \\
\hline $65-74$ & $\begin{array}{l}8930 \\
(20.1)\end{array}$ & $1448(7.8)$ & & $\begin{array}{l}6015 \\
(16.2)\end{array}$ & $\begin{array}{l}4363 \\
(16.8)\end{array}$ & & $\begin{array}{l}9296 \\
(18.9)\end{array}$ & $\begin{array}{l}1082 \\
(7.7)\end{array}$ & & $\begin{array}{l}7799 \\
(17.3)\end{array}$ & $\begin{array}{l}2579 \\
(14.4)\end{array}$ & & $\begin{array}{l}8755 \\
(16.6)\end{array}$ & $\begin{array}{l}1623 \\
(15.5)\end{array}$ & \\
\hline$\geqq 75$ & $\begin{array}{l}7647 \\
(17.2)\end{array}$ & $1211(6.5)$ & & $\begin{array}{l}5084 \\
(13.7)\end{array}$ & $\begin{array}{l}3774 \\
(14.5)\end{array}$ & & $\begin{array}{l}7937 \\
(16.2)\end{array}$ & $921(6.6)$ & & $\begin{array}{l}6348 \\
(14.1)\end{array}$ & $\begin{array}{l}2510 \\
(14.0)\end{array}$ & & $\begin{array}{l}7350 \\
(14.0)\end{array}$ & $\begin{array}{l}1508 \\
(14.4)\end{array}$ & \\
\hline \multicolumn{16}{|l|}{ Drinking status ( $n=62343$ ) } \\
\hline Once or more per month & $\begin{array}{l}17711 \\
(40.3)\end{array}$ & $\begin{array}{l}10023 \\
(54.4)\end{array}$ & $<0.001$ & $\begin{array}{l}15582 \\
(42.6)\end{array}$ & $\begin{array}{l}12152 \\
(47.2)\end{array}$ & $<0.001$ & $\begin{array}{l}21409 \\
(44.2)\end{array}$ & $\begin{array}{l}6325 \\
(45.6)\end{array}$ & $<0.001$ & $\begin{array}{l}19427 \\
(43.5)\end{array}$ & $\begin{array}{l}8307 \\
(47.0)\end{array}$ & $<0.001$ & $\begin{array}{l}21910 \\
(42.1)\end{array}$ & $\begin{array}{l}5824 \\
(56.3)\end{array}$ & $<0.001$ \\
\hline \multicolumn{16}{|c|}{ Smoking status $(\mathrm{n}=61933)$} \\
\hline Current & $\begin{array}{l}6661 \\
(15.3)\end{array}$ & $\begin{array}{l}6741 \\
(36.8)\end{array}$ & $<0.001$ & $\begin{array}{l}7447 \\
(20.5)\end{array}$ & $\begin{array}{l}5955 \\
(23.3)\end{array}$ & $<0.001$ & $\begin{array}{l}9247 \\
(19.2)\end{array}$ & $\begin{array}{l}4155 \\
(30.1)\end{array}$ & $<0.001$ & $\begin{array}{l}8435 \\
(19.0)\end{array}$ & $\begin{array}{l}4967 \\
(28.3)\end{array}$ & $<0.001$ & $\begin{array}{l}9660 \\
(18.7)\end{array}$ & $\begin{array}{l}3742 \\
(36.3)\end{array}$ & $<0.001$ \\
\hline \multicolumn{16}{|c|}{ Health condition ( $n=62009)$} \\
\hline Poor/very poor & $\begin{array}{l}8373 \\
(19.1) \\
-(239)\end{array}$ & $\begin{array}{l}3174 \\
(17.3)\end{array}$ & $<0.001$ & $\begin{array}{l}6576 \\
(18.1)\end{array}$ & $\begin{array}{l}4971 \\
(19.4)\end{array}$ & $<0.001$ & $\begin{array}{l}8849 \\
(18.4)\end{array}$ & $\begin{array}{l}2698 \\
(19.6)\end{array}$ & $<0.001$ & $\begin{array}{l}8025 \\
(18.1)\end{array}$ & $\begin{array}{l}3522 \\
(20.0)\end{array}$ & $<0.001$ & $\begin{array}{l}9528 \\
(18.5)\end{array}$ & $\begin{array}{l}2019 \\
(19.6)\end{array}$ & $<0.001$ \\
\hline \multicolumn{16}{|c|}{ History of mental illness ( $n=61239$ ) } \\
\hline Yes & $2370(5.5)$ & $1036(5.8)$ & 0.10 & $1959(5.5)$ & $\begin{array}{l}1447 \\
(5.7)\end{array}$ & 0.08 & $\begin{array}{l}2542 \\
(5.3)\end{array}$ & $864(6.4)$ & $<0.001$ & $2403(5.5)$ & $1003(5.8)$ & 0.07 & $2770(5.4)$ & $636(6.3)$ & $<0.01$ \\
\hline \multicolumn{16}{|c|}{ History of other illness ( $n=63047$ ) } \\
\hline Yes & $\begin{array}{l}44428 \\
(99.8)\end{array}$ & $\begin{array}{l}18484 \\
(99.8)\end{array}$ & 0.14 & $\begin{array}{l}36954 \\
(99.8)\end{array}$ & $\begin{array}{l}25958 \\
(99.8)\end{array}$ & 0.05 & $\begin{array}{l}48984 \\
(99.8)\end{array}$ & $\begin{array}{l}13928 \\
(99.7)\end{array}$ & 0.06 & $\begin{array}{l}45089 \\
(99.8)\end{array}$ & $\begin{array}{l}17823 \\
(99.8)\end{array}$ & 0.16 & $\begin{array}{l}52487 \\
(99.8)\end{array}$ & $\begin{array}{l}10425 \\
(99.8)\end{array}$ & 0.49 \\
\hline \multicolumn{16}{|c|}{ Leisure-time physical activity $(n=62150)$} \\
\hline Almost never & $\begin{array}{l}20960 \\
(47.9)\end{array}$ & $\begin{array}{l}12100 \\
(65.9)\end{array}$ & $<0.001$ & $\begin{array}{l}14597 \\
(56.8)\end{array}$ & $\begin{array}{l}18463 \\
(50.6)\end{array}$ & $<0.001$ & $\begin{array}{l}23962 \\
(49.6)\end{array}$ & $\begin{array}{l}9098 \\
(65.6)\end{array}$ & $<0.001$ & $\begin{array}{l}22488 \\
(50.5)\end{array}$ & $\begin{array}{l}10572 \\
(59.9)\end{array}$ & $<0.001$ & $\begin{array}{l}26734 \\
(51.6)\end{array}$ & $\begin{array}{l}6326 \\
(61.3)\end{array}$ & $<0.001$ \\
\hline \multicolumn{16}{|c|}{ Sleeping habits $(n=52474)$} \\
\hline $\begin{array}{l}\text { Very dissatisfied or have } \\
\text { not slept at all } \\
\text { Educational attainment }(n=6\end{array}$ & $\begin{array}{l}1502(4.1) \\
0998)\end{array}$ & $752(4.7)$ & $<0.001$ & $1282(4.2)$ & $\begin{array}{l}972 \\
(4.5)\end{array}$ & $<0.01$ & $\begin{array}{l}1644 \\
(4.1)\end{array}$ & $610(5.1)$ & $<0.001$ & $1512(4.0)$ & $742(5.0)$ & $<0.001$ & $1815(4.1)$ & $439(5.1)$ & $<0.001$ \\
\hline $\begin{array}{l}\text { University/graduate } \\
\text { school } \\
\text { Experiences of the Great Ec } \\
\text { Earthquake }(\mathrm{n}=63047)\end{array}$ & $\begin{array}{l}3809(8.9) \\
\text { ast Japan Ea }\end{array}$ & $\begin{array}{l}1648(9.1) \\
\text { arthquake }\end{array}$ & $<0.001$ & $3310(9.2)$ & $\begin{array}{l}2147 \\
(8.5)\end{array}$ & $<0.001$ & $\begin{array}{l}4076 \\
(8.6)\end{array}$ & $\begin{array}{l}1381 \\
(10.2)\end{array}$ & $<0.001$ & $4197(9.6)$ & $1260(7.3)$ & $<0.001$ & $4643(9.1)$ & $814(8.1)$ & $<0.001$ \\
\hline Yes & $\begin{array}{l}43040 \\
(96.7)\end{array}$ & $\begin{array}{l}17974 \\
(97.0)\end{array}$ & 0.02 & $\begin{array}{l}35806 \\
(96.7)\end{array}$ & $\begin{array}{l}25208 \\
(96.9)\end{array}$ & 0.03 & $\begin{array}{l}47487 \\
(96.8)\end{array}$ & $\begin{array}{l}13527 \\
(96.9)\end{array}$ & 0.28 & $\begin{array}{l}43737 \\
(96.8)\end{array}$ & $\begin{array}{l}17277 \\
(96.7)\end{array}$ & 0.25 & $\begin{array}{l}50952 \\
(96.9)\end{array}$ & $\begin{array}{l}10062 \\
(96.3)\end{array}$ & $<0.01$ \\
\hline Tsunami $(n=63$ 047) & & & & & & & & & & & & & & & \\
\hline Yes & $\begin{array}{l}8762 \\
(19.7)\end{array}$ & $\begin{array}{l}3673 \\
(19.8)\end{array}$ & 0.35 & $\begin{array}{l}7523 \\
(20.3)\end{array}$ & $\begin{array}{l}4912 \\
(18.9)\end{array}$ & $<0.001$ & $\begin{array}{l}9737 \\
(19.8)\end{array}$ & $\begin{array}{l}2698 \\
(19.3)\end{array}$ & 0.09 & $\begin{array}{l}8985 \\
(19.9)\end{array}$ & $\begin{array}{l}3450 \\
(19.7)\end{array}$ & 0.05 & $\begin{array}{l}10368 \\
(19.7)\end{array}$ & $\begin{array}{l}2067 \\
(19.8)\end{array}$ & 0.44 \\
\hline
\end{tabular}




\begin{tabular}{|c|c|c|c|c|c|c|c|c|c|c|c|c|c|c|c|}
\hline & \multicolumn{3}{|c|}{ Fruits (non-juice) } & \multicolumn{3}{|l|}{ Fruit juice } & \multicolumn{3}{|c|}{ Soya bean products } & \multicolumn{3}{|l|}{ Milk } & \multicolumn{3}{|c|}{$\begin{array}{l}\text { Yogurt and lactobacillus } \\
\text { drinks }\end{array}$} \\
\hline & $\begin{array}{l}1-2 \\
\text { times/ } \\
\text { week or } \\
\text { more }\end{array}$ & $\begin{array}{l}\text { Less than } \\
\text { once/week } \\
\text { or never }\end{array}$ & $\begin{array}{l}\text { p } \\
\text { value* }\end{array}$ & $\begin{array}{l}\text { Less than } \\
\text { once/week } \\
\text { or more }\end{array}$ & Never & $\begin{array}{l}\text { p } \\
\text { value* }\end{array}$ & Daily & $\begin{array}{l}5-6 \\
\text { times/ } \\
\text { week or } \\
\text { less }\end{array}$ & $\begin{array}{l}\text { p } \\
\text { value* }\end{array}$ & $\begin{array}{l}1-2 \\
\text { times/ } \\
\text { week or } \\
\text { more }\end{array}$ & $\begin{array}{l}\text { Less than } \\
\text { once/week } \\
\text { or never }\end{array}$ & $\begin{array}{l}\text { p } \\
\text { value* }^{*}\end{array}$ & $\begin{array}{l}1-2 \\
\text { times/ } \\
\text { week or } \\
\text { more }\end{array}$ & $\begin{array}{l}\text { Less than } \\
\text { once/week } \\
\text { or never }\end{array}$ & $\begin{array}{l}p \\
\text { value }\end{array}$ \\
\hline \multicolumn{16}{|c|}{ Nuclear reactor accident ( $n=63$ 047) } \\
\hline Yes & $\begin{array}{l}23569 \\
(52.9)\end{array}$ & $\begin{array}{l}9277 \\
(50.1)\end{array}$ & $<0.001$ & $\begin{array}{l}19313 \\
(52.1)\end{array}$ & $\begin{array}{l}13533 \\
(52.0)\end{array}$ & 0.41 & $\begin{array}{l}25731 \\
(52.4)\end{array}$ & $\begin{array}{l}7115 \\
(50.9)\end{array}$ & 0.001 & $\begin{array}{l}23362 \\
(51.7)\end{array}$ & $\begin{array}{l}9484 \\
(53.1)\end{array}$ & $<0.001$ & $\begin{array}{l}27359 \\
(52.0)\end{array}$ & $\begin{array}{l}5487 \\
(52.5)\end{array}$ & 0.18 \\
\hline \multicolumn{16}{|l|}{ None $(n=63047)$} \\
\hline Yes & $509(1.1)$ & $187(1.0)$ & 0.08 & $428(1.2)$ & $\begin{array}{l}268 \\
(1.0)\end{array}$ & 0.08 & $\begin{array}{l}557 \\
(1.1)\end{array}$ & $139(1.0)$ & 0.09 & $518(1.1)$ & $178(1.0)$ & 0.06 & $570(1.1)$ & $126(1.2)$ & 0.15 \\
\hline \multicolumn{16}{|c|}{ Living arrangements ( $n=63047$ ) } \\
\hline $\begin{array}{l}\text { Evacuation shelter or } \\
\text { temporary housing }\end{array}$ & $\begin{array}{l}4743 \\
(10.7)\end{array}$ & $\begin{array}{l}1856 \\
(10.0)\end{array}$ & $<0.01$ & $\begin{array}{l}2664 \\
(10.2)\end{array}$ & $\begin{array}{l}3935 \\
(10.6)\end{array}$ & 0.70 & $\begin{array}{l}5153 \\
(10.5)\end{array}$ & $\begin{array}{l}1446 \\
(10.4)\end{array}$ & 0.32 & $\begin{array}{l}4579 \\
(10.1)\end{array}$ & $\begin{array}{l}2020 \\
(11.3)\end{array}$ & $<0.001$ & $\begin{array}{l}5441 \\
(10.3)\end{array}$ & $\begin{array}{l}1158 \\
(11.1)\end{array}$ & 0.01 \\
\hline \multicolumn{16}{|c|}{ Changes in work situation $(n=60077)$} \\
\hline Yes & $\begin{array}{l}21856 \\
(51.9)\end{array}$ & $\begin{array}{l}10262 \\
(57.1)\end{array}$ & $<0.001$ & $\begin{array}{l}18994 \\
(53.9)\end{array}$ & $\begin{array}{l}13124 \\
(52.9)\end{array}$ & $<0.01$ & $\begin{array}{l}24406 \\
(52.3)\end{array}$ & $\begin{array}{l}7712 \\
(57.3)\end{array}$ & $<0.001$ & $\begin{array}{l}22826 \\
(53.1)\end{array}$ & $\begin{array}{l}9292 \\
(54.5)\end{array}$ & $<0.001$ & $\begin{array}{l}26755 \\
(53.5)\end{array}$ & $\begin{array}{l}5363 \\
(53.4)\end{array}$ & 0.46 \\
\hline \multicolumn{16}{|c|}{ Loss of employment $(n=63047)$} \\
\hline & $\begin{array}{l}8922 \\
(20.0)\end{array}$ & $\begin{array}{l}3780 \\
(20.4)\end{array}$ & 0.16 & $\begin{array}{l}7380 \\
(19.9)\end{array}$ & $\begin{array}{l}5322 \\
(20.5)\end{array}$ & 0.05 & $\begin{array}{l}9691 \\
(19.7)\end{array}$ & $\begin{array}{l}3011 \\
(21.6)\end{array}$ & $<0.001$ & $\begin{array}{l}9106 \\
(20.2)\end{array}$ & $\begin{array}{l}3596 \\
(20.1)\end{array}$ & 0.47 & $\begin{array}{l}10768 \\
(20.5)\end{array}$ & $\begin{array}{l}1934 \\
(18.5)\end{array}$ & $<0.001$ \\
\hline \multicolumn{16}{|c|}{ Decreased income $(n=63047)$} \\
\hline Yes & $\begin{array}{l}7562 \\
(17.0)\end{array}$ & $\begin{array}{l}3951 \\
(21.3)\end{array}$ & $<0.001$ & $\begin{array}{l}6856 \\
(18.5)\end{array}$ & $\begin{array}{l}4657 \\
(17.9)\end{array}$ & 0.03 & $\begin{array}{l}8735 \\
(17.8)\end{array}$ & $\begin{array}{l}2778 \\
(19.9)\end{array}$ & $<0.001$ & $\begin{array}{l}8075 \\
(17.9)\end{array}$ & $\begin{array}{l}3438 \\
(19.2)\end{array}$ & $<0.001$ & $\begin{array}{l}9402 \\
(17.9)\end{array}$ & $\begin{array}{l}2111 \\
(20.2)\end{array}$ & $<0.001$ \\
\hline \multicolumn{16}{|c|}{ Death of someone close ( $n=62081)$} \\
\hline Yes & $\begin{array}{l}8788 \\
(20.1)\end{array}$ & $\begin{array}{l}3218 \\
(17.6)\end{array}$ & $<0.001$ & $\begin{array}{l}7198 \\
(19.8)\end{array}$ & $\begin{array}{l}4808 \\
(18.7)\end{array}$ & 0.001 & $\begin{array}{l}9466 \\
(19.6)\end{array}$ & $\begin{array}{l}2540 \\
(18.4)\end{array}$ & 0.001 & $\begin{array}{l}8787 \\
(19.8)\end{array}$ & $\begin{array}{l}3219 \\
(18.3)\end{array}$ & $<0.001$ & $\begin{array}{l}10220 \\
(19.7)\end{array}$ & $\begin{array}{l}1786 \\
(17.4)\end{array}$ & $<0.001$ \\
\hline
\end{tabular}




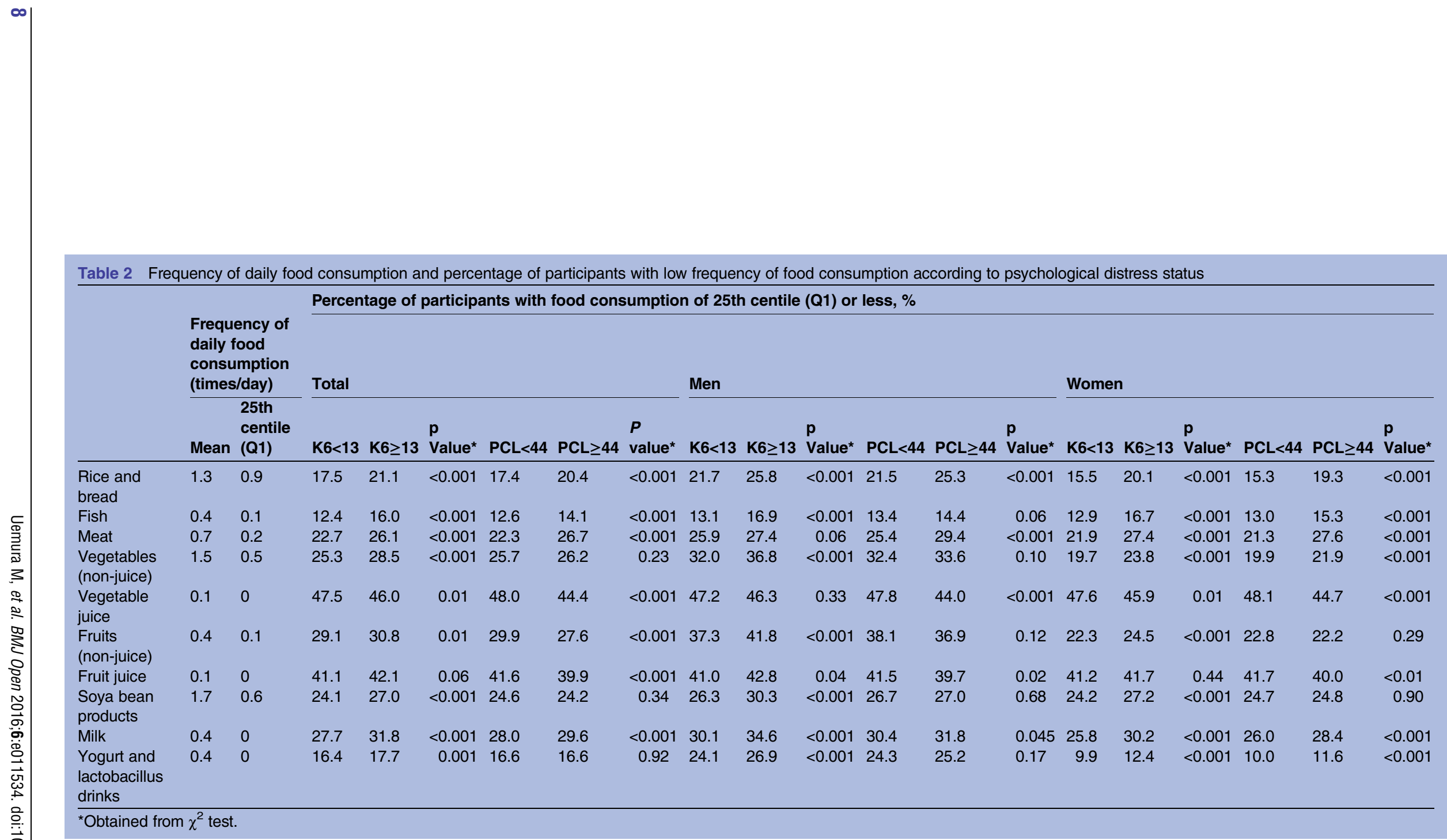

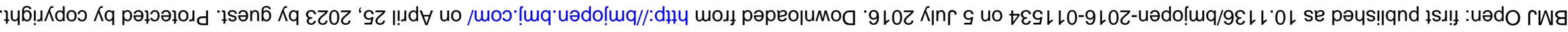


Table 3 ORs and $95 \%$ Cls for low frequency of food consumption according to the K6 score

\begin{tabular}{|c|c|c|c|c|c|c|c|}
\hline & \multirow{2}{*}{$\begin{array}{l}\text { Participants with } \\
\text { low-frequency consumption } \\
\text { within group, } \mathrm{n} / \mathrm{N}(\%)\end{array}$} & \multicolumn{2}{|l|}{ Total } & \multicolumn{2}{|l|}{ Men } & \multicolumn{2}{|l|}{ Women } \\
\hline & & Model 1 & Model 2 & Model 1 & Model 2 & Model 1 & Model 2 \\
\hline \multicolumn{8}{|c|}{ Rice and bread } \\
\hline$K 6<13$ & $9850 / 56151(17.5)$ & 1 (reference) & & 1 (reference) & & 1 (reference) & \\
\hline$K 6 \geq 13$ & $2047 / 9715(21.1)$ & 1.31 (1.24 to 1.39$)$ & 1.16 (1.08 to 1.25$)$ & 1.25 (1.15 to 1.36$)$ & 1.14 (1.02 to 1.28$)$ & $1.36(1.27$ to 1.46$)$ & 1.16 (1.05 to 1.28$)$ \\
\hline \multicolumn{8}{|c|}{ 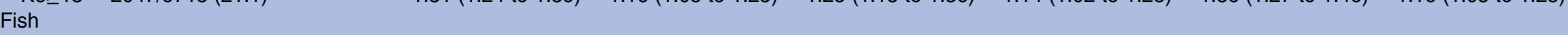 } \\
\hline$K 6<13$ & 6984/56 151 (12.4) & 1 (reference) & & 1 (reference) & & 1 (reference) & \\
\hline$K 6 \geq 13$ & $1550 / 9715(16.0)$ & $1.43(1.35$ to 1.52$)$ & $1.22(1.12$ to 1.32$)$ & 1.37 (1.24 to 1.51$)$ & $1.17(1.03$ to 1.34$)$ & $1.47(1.36$ to 1.59$)$ & $1.24(1.12$ to 1.39$)$ \\
\hline \multicolumn{8}{|l|}{ Meat } \\
\hline$K 6<13$ & $12756 / 56151(22.7)$ & 1 (reference) & & 1 (reference) & & 1 (reference) & \\
\hline$K 6 \geq 13$ & $2531 / 9715(26.1)$ & $1.20(1.14$ to 1.26$)$ & 1.09 (1.02 to 1.17$)$ & 1.09 (1.00 to 1.18$)$ & 0.99 (0.88 to 1.11$)$ & 1.27 (1.19 to 1.36$)$ & 1.16 (1.06 to 1.28$)$ \\
\hline \multicolumn{8}{|c|}{ Vegetables (non-juice) } \\
\hline $\mathrm{K} 6<13$ & $13611 / 56151(25.3)$ & 1 (reference) & & 1 (reference) & & 1 (reference) & \\
\hline$K 6 \geq 13$ & $2637 / 9715(28.5)$ & $1.32(1.26$ to 1.39$)$ & $1.12(1.04$ to 1.20$)$ & $1.26(1.17$ to 1.36$)$ & 1.04 (0.94 to 1.16$)$ & $1.36(1.27$ to 1.46$)$ & $1.19(1.08$ to 1.31$)$ \\
\hline \multicolumn{8}{|c|}{ Vegetable juice } \\
\hline$K 6<13$ & $25532 / 56151(47.5)$ & 1 (reference) & & 1 (reference) & & 1 (reference) & \\
\hline$K 6 \geq 13$ & $4256 / 9715(46.0)$ & $0.94(0.90$ to 0.99$)$ & $1.00(0.95$ to 1.07$)$ & 0.96 (0.90 to 1.04$)$ & 1.03 (0.93 to 1.14$)$ & 0.94 (0.88 to 0.99$)$ & 0.99 (0.91 to 1.07$)$ \\
\hline \multicolumn{8}{|c|}{ Fruits (non-juice) } \\
\hline$K 6<13$ & 15 680/56 $151(29.1)$ & 1 (reference) & & 1 (reference) & & 1 (reference) & \\
\hline$K 6 \geq 13$ & $2850 / 9715(30.8)$ & $1.26(1.20$ to 1.32$)$ & $1.13(1.05$ to 1.21$)$ & 1.24 (1.14 to 1.34$)$ & 1.11 (0.99 to 1.23$)$ & $1.27(1.19$ to 1.36$)$ & $1.14(1.04$ to 1.25$)$ \\
\hline \multicolumn{8}{|l|}{ Fruit juice } \\
\hline$K 6<13$ & 22 110/56 151 (41.1) & 1 (reference) & & 1 (reference) & & 1 (reference) & \\
\hline$K 6 \geq 13$ & $3894 / 9715(42.1)$ & $1.03(0.99$ to 1.08$)$ & $1.03(0.96$ to 1.09$)$ & $1.08(1.00$ to 1.16$)$ & $1.10(0.99$ to 1.21$)$ & $1.00(0.94$ to 1.06$)$ & 0.98 (0.91 to 1.06$)$ \\
\hline \multicolumn{8}{|c|}{ Soya bean products } \\
\hline $\mathrm{K} 6<13$ & $13524 / 56151(24.1)$ & 1 (reference) & & 1 (reference) & & 1 (reference) & \\
\hline$K 6 \geq 13$ & $2619 / 9715(27.0)$ & 1.28 (1.22 to 1.35$)$ & 1.08 (1.01 to 1.16$)$ & 1.25 (1.15 to 1.35$)$ & 0.99 (0.89 to 1.11$)$ & 1.29 (1.21 to 1.38$)$ & $1.13(1.03$ to 1.24$)$ \\
\hline \multicolumn{8}{|l|}{ Milk } \\
\hline$K 6<13$ & 14 930/56 $151(27.7)$ & 1 (reference) & & 1 (reference) & & 1 (reference) & \\
\hline$K 6 \geq 13$ & $2937 / 9715(31.8)$ & $1.24(1.18$ to 1.30$)$ & $1.18(1.10$ to 1.26$)$ & 1.23 (1.14 to 1.33$)$ & 1.21 (1.09 to 1.34$)$ & 1.24 (1.16 to 1.32$)$ & 1.15 (1.05 to 1.25$)$ \\
\hline \multicolumn{8}{|c|}{ Yogurt and lactobacillus drinks } \\
\hline $\mathrm{K} 6<13$ & $8811 / 56151(16.4)$ & 1 (reference) & & 1 (reference) & & 1 (reference) & \\
\hline$K 6 \geq 13$ & $1637 / 9715(17.7)$ & $1.22(1.15$ to 1.29$)$ & $1.23(1.13$ to 1.34$)$ & $1.17(1.07$ to 1.27$)$ & $1.20(1.07$ to 1.34$)$ & 1.27 (1.17 to 1.39$)$ & $1.27(1.12$ to 1.43$)$ \\
\hline
\end{tabular}

$\mathrm{n}$, indicates number in group; $\mathrm{N}$, number of participants.

Model 1: Adjusted for age and sex.

Model 2: Model 1+smoking status, drinking status, perceived health condition, history of mental illness, history of other illness, leisure-time physical activity, sleeping habits, educational

attainment, experiences of the Great East Japan Earthquake, living arrangements, changes in work situation, loss of employment, decreased income and death of someone close. 
(1.01 to 1.16$), 1.18$ (1.10 to 1.26$), 1.23$ (1.13 to 1.34 ), respectively (model 2 ). In the analysis stratified by sex, these positive associations between non-specific mental health distress and low frequency of food consumption were predominantly observed in women (all $\mathrm{p}<0.05$ ) (model 2).

In the age-adjusted logistic model, traumatic symptoms were positively associated with a low intake frequency for rice and bread, fish, meat, vegetables (non-juice), fruits (non-juice), soya bean products, milk and yogurt or lactobacillus drinks (model 1) (table 4). On the other hand, traumatic symptoms were inversely associated with low frequencies of vegetable juice and fruit juice intake. In the multivariable adjusted model, these associations were replicated for rice and bread, fish, meat, vegetables (non-juice), vegetable juice, fruit juice, milk and yogurt or lactobacillus drinks 1.13 (1.06 to 1.21 ), 1.14 (1.07 to 1.22 ), 1.07 (1.01 to 1.15 ), 1.07 (1.00 to 1.14$), 0.92$ (0.88 to 0.98$), 0.91$ (0.86 to 0.96$), 1.07$ (1.01 to 1.13 ), 1.14 (1.06 to 1.23$)$, respectively; however, no significant associations for fruits (non-juice) and soya bean products were observed (model 2). In the analysis stratified by sex, these associations between traumatic symptoms and frequency of food consumption were predominantly observed in women $($ all $\mathrm{p}<0.05)$ (model 2$)$.

\section{DISCUSSION}

In data from the baseline survey of evacuees of the Great East Japan Earthquake, psychological distress was significantly associated with a low intake frequency for certain foods, including rice and bread, fish, meat, vegetables (non-juice) and dairy products, after adjustment for a number of potentially confounding lifestyle-related and disaster-related variables. These associations were predominantly observed in women.

One previous baseline study from Iwate Prefecture reported that participants in difficult living conditions were likely to have unhealthy dietary patterns and lower intake frequency of certain foods. ${ }^{16}$ Another study suggested that evacuees living in shelters experience difficulties in obtaining and preparing meals due to shortages of cooking equipment/utilities and of food generally. ${ }^{17}$ However, we found a novel indication that the associations between psychological distress and low frequency of intake of certain foods were independent of several confounding factors, including disaster-related factors like living arrangements, predominantly in women. After a stratified analysis by living arrangement, the association between psychological distress and dietary intake was essentially the same between evacuees living in evacuation shelters or temporary housing and evacuees living in rental housing, relatives' homes or their own home (see online supplementary tables S1 and S2). In the same way, as a result of the stratified analysis by socioeconomic conditions, the aforementioned association was essentially the same among all evacuees regardless of whether they had suffered from decreased income or not (see online supplementary tables S3 and S4). Thus, the observed low frequency of food consumption could have been caused by psychological distress. However, the low intake frequency of certain foods also could be caused by unexamined factors. For instance, an inability to grow or ingest local Fukushima products due to concerns over radiation contamination could be regarded as a major cause for the low intake frequency of certain foods. ${ }^{18}$ Furthermore, other unexamined factors, such as limited accessibility of certain foods, limited affordability and low availability, might have caused low intake frequency of certain foods.

The associations between non-specific mental health distress and the low frequencies for various food groups (refer to table 3) are in accordance with previous studies that carefully tracked dietary patterns of the same food items. $^{19} 20$ However, the associations between nonspecific mental health distress and low frequency of intake of meat, vegetables (non-juice), fruits (non-juice) and soya bean products were found only in women. The associations in men disappeared after adjustment for health condition, lifestyle-related and disaster-related factors (refer to model 2 in table 3), which can be interpreted as poor health conditions, lifestyle-related and disaster-related factors causing low frequency of intake of such foods in men. Participants with psychological distress might have lost the motivation to cook or to pay careful attention to their own health by taking meals, as in Japan this task is traditionally carried out more frequently by women. This might be the reason that the association between psychological distress and dietary intake independent from health condition, lifestyle-related and disaster-related factors was predominantly observed in women. In addition, associations between depression and some nutrients such as vitamin B (including folate) and n-3 polyunsaturated fatty acids (n-3PUFAs) have been reported. ${ }^{21} 22$ Therefore, our observed associations between non-specific mental health distress and low intake frequency of fish and vegetables align well with the results of previous studies. Especially interesting against the background of high mental distress and low frequency of dietary intake are the associations between high traumatic symptoms and high consumption of vegetable and fruit juices observed in women in this study. This might be attributable to the fact that vegetable and fruit juices are readily available for consumption with little preparation, which is more favourable from the viewpoint of women, who have more opportunities to cook in Japanese culture. More frequent juice intake may have led to weight gain and even diabetes among evacuees. ${ }^{12}$

From a different viewpoint, participants who did not habitually eat fish, vegetables and probiotic products before the disaster may have been more likely to be in worse mental health condition than participants who did. Previous cohort studies reported that high intake of fish containing n-3PUFAs, ${ }^{10}$ vegetables containing vitamin $\mathrm{B},{ }^{10}$ probiotic products ${ }^{23}$ and a diverse diet rich 
Table 4 ORs and $95 \%$ Cls for low frequency of food consumption according to PCL score

\begin{tabular}{|c|c|c|c|c|c|c|c|}
\hline & \multirow{2}{*}{$\begin{array}{l}\text { Participants with } \\
\text { low-frequency consumption } \\
\text { within group, } \mathrm{n} / \mathrm{N}(\%)\end{array}$} & \multicolumn{2}{|l|}{ Total } & \multicolumn{2}{|l|}{ Men } & \multicolumn{2}{|l|}{ Women } \\
\hline & & Model 1 & Model 2 & Model 1 & Model 2 & Model 1 & Model 2 \\
\hline \multicolumn{8}{|c|}{ Rice and bread } \\
\hline $\mathrm{PCL}<44$ & 9059/51934 (17.4) & 1 (reference) & & 1 (reference) & & 1 (reference) & \\
\hline$P C L \geq 44$ & 2838/13 932 (20.4) & 1.27 (1.21 to 1.33$)$ & $1.13(1.06$ to 1.21$)$ & 1.23 (1.14 to 1.32$)$ & 1.10 (1.00 to 1.22$)$ & 1.31 (1.23 to 1.40$)$ & 1.15 (1.05 to 1.26$)$ \\
\hline \multicolumn{8}{|l|}{ Fish } \\
\hline $\mathrm{PCL}<44$ & $6564 / 51934(12.6)$ & 1 (reference) & & 1 (reference) & & 1 (reference) & \\
\hline$P C L \geq 44$ & $1970 / 13932(14.1)$ & $1.31(1.24$ to 1.39$)$ & 1.14 (1.07 to 1.22$)$ & $1.22(1.11$ to 1.33$)$ & $1.10(0.98$ to 1.25$)$ & $1.37(1.28$ to 1.47$)$ & $1.24(1.12$ to 1.37$)$ \\
\hline \multicolumn{8}{|l|}{ Meat } \\
\hline $\mathrm{PCL}<44$ & $11561 / 51934(22.3)$ & 1 (reference) & & 1 (reference) & & 1 (reference) & \\
\hline$P C L \geq 44$ & $3726 / 13932(26.7)$ & $1.22(1.17$ to 1.28$)$ & 1.07 (1.01 to 1.15$)$ & $1.13(1.06$ to 1.22$)$ & $1.13(1.02$ to 1.24$)$ & $1.28(1.21$ to 1.36$)$ & 1.15 (1.06 to 1.25$)$ \\
\hline \multicolumn{8}{|c|}{ Vegetables (non-juice) } \\
\hline $\mathrm{PCL}<44$ & $12797 / 51934(25.7)$ & 1 (reference) & & 1 (reference) & & 1 (reference) & \\
\hline $\mathrm{PCL} \geq 44$ & $3451 / 13932(26.2)$ & $1.23(1.18$ to 1.29$)$ & $1.07(1.00$ to 1.14$)$ & $1.20(1.12$ to 1.29$)$ & $1.04(0.95$ to 1.15$)$ & $1.26(1.18$ to 1.34$)$ & $1.10(1.01$ to 1.20$)$ \\
\hline \multicolumn{8}{|c|}{ Vegetable juice } \\
\hline $\mathrm{PCL}<44$ & $23931 / 51934$ (48.0) & 1 (reference) & & 1 (reference) & & 1 (reference) & \\
\hline$P C L \geq 44$ & $5857 / 13932(44.4)$ & $0.87(0.84$ to 0.91$)$ & $0.92(0.88$ to 0.98$)$ & $0.86(0.80$ to 0.91$)$ & $0.92(0.85$ to 1.00$)$ & $0.88(0.84$ to 0.93$)$ & 0.93 (0.87 to 0.997$)$ \\
\hline \multicolumn{8}{|c|}{ Fruits (non-juice) } \\
\hline $\mathrm{PCL}<44$ & $14886 / 51934(29.9)$ & 1 (reference) & & 1 (reference) & & 1 (reference) & \\
\hline$P C L \geq 44$ & $3644 / 13932(27.6)$ & $1.13(1.08$ to 1.19$)$ & $1.04(0.97$ to 1.10$)$ & $1.12(1.04$ to 1.20$)$ & $1.06(0.97$ to 1.17$)$ & 1.15 (1.08 to 1.22$)$ & $1.02(0.94$ to 1.12$)$ \\
\hline \multicolumn{8}{|l|}{ Fruit juice } \\
\hline $\mathrm{PCL}<44$ & 20 747/51 934 (41.6) & 1 (reference) & & 1 (reference) & & 1 (reference) & \\
\hline$P C L \geq 44$ & $5257 / 13932(39.9)$ & $0.91(0.87$ to 0.95$)$ & $0.91(0.86$ to 0.96$)$ & $0.91(0.86$ to 0.97$)$ & 0.95 (0.97 to 1.03$)$ & $0.91(0.86$ to 0.95$)$ & $0.89(0.83$ to 0.95$)$ \\
\hline \multicolumn{8}{|c|}{ Soya bean products } \\
\hline $\mathrm{PCL}<44$ & $12772 / 51934(24.6)$ & 1 (reference) & & 1 (reference) & & 1 (reference) & \\
\hline$P C L \geq 44$ & $3371 / 13932(24.2)$ & $1.17(1.12$ to 1.23$)$ & $1.07(1.00$ to 1.14$)$ & $1.18(1.10$ to 1.27$)$ & $1.00(0.90$ to 1.10$)$ & $1.16(1.10$ to 1.23$)$ & $0.99(0.91$ to 1.08$)$ \\
\hline \multicolumn{8}{|l|}{ Milk } \\
\hline $\mathrm{PCL}<44$ & 13 960/51 $934(28.0)$ & 1 (reference) & & 1 (reference) & & 1 (reference) & \\
\hline$P C L \geq 44$ & $3907 / 13932(29.6)$ & $1.12(1.08$ to 1.17$)$ & 1.07 (1.01 to 1.13$)$ & $1.11(1.04$ to 1.18$)$ & $1.13(1.03$ to 1.23$)$ & $1.13(1.07$ to 1.19$)$ & $1.03(0.95$ to 1.11$)$ \\
\hline \multicolumn{8}{|c|}{ Yogurt and lactobacillus drinks } \\
\hline $\mathrm{PCL}<44$ & $8260 / 51934(16.6)$ & 1 (reference) & & 1 (reference) & & 1 (reference) & \\
\hline$P C L \geq 44$ & $2188 / 13932(16.6)$ & $1.11(1.05$ to 1.17$)$ & $1.14(1.06$ to 1.23$)$ & $1.07(1.00$ to 1.15$)$ & $1.16(1.05$ to 1.28$)$ & $1.16(1.07$ to 1.26$)$ & $1.13(1.01$ to 1.27$)$ \\
\hline
\end{tabular}


in vegetables, fruits, soya bean products, mushrooms and green tea $^{24}$ is associated with reduced depression risk. Similarly, high glycaemic index (GI) diets could be a risk factor for depression. ${ }^{25}$ Although our finding of a low frequency of rice and bread intake correlating with high psychological distress is not in line with this previous result, it does not contradict it. It is not improbable that participants who had a low frequency of rice and bread intake tended to eat ready-to-eat foods, which are often foods with high GI. An investigation of causal correlation in a cohort study of evacuees would thus obtain valuable findings contributing to the prevention and improvement of psychological distress.

Although it is unclear why the results differed by sex, this study has provided important findings. Associations between psychological distress and dietary intake were shown for the first time in evacuees from a major disaster. Therefore, our findings could promote future study as well as serve as reference when preventing or remedying the mental health problems of evacuees.

This study has several limitations. First, the overall response rate was low $(40.7 \%)$, and there was a relatively high percentage of missing observations in the psychological distress category (10.3\%). It should be noted that participants in this study were not always representative of all the residents in Fukushima, and they could enhance or weaken the association between psychological distress and dietary intake. Second, in the respondents to the questionnaire, the $9.3 \%$ who responded by proxy were included and this may affect the results. However, in the analysis covering only those who responded in person, almost the same associations between psychological distress and dietary intake were identified, with the exceptions of the association between non-specific mental health distress and fruit juice in men, and traumatic symptoms and vegetable juice in all participants (model 2 in online supplementary tables S5 and S6). Third, we used an FFQ of 19 items, in which some food items consumed by modern Japanese people, such as processed food, fast food, ready-to-eat foods and recently introduced Western foods, were not included. Since evacuees with a low intake frequency of several fresh foods may compensate with these foods, the associations between psychological distress and these foods must be investigated in our further research. Finally, we were not able to calculate either the quantity of each food or participants' dietary nutrient intake, because we had no information on portion sizes. We cannot therefore conclude that the evacuees actually ate less quantity of the foods we have reported here.

In conclusion, non-specific mental health distress among evacuees after the Great East Japan Earthquake was associated with a low intake frequency of certain foods, such as rice and bread, fish, meat, vegetables (non-juice), fruit (non-juice), soya bean products, milk and yogurt and lactobacillus drinks. In an analogous fashion, traumatic symptoms were associated with a low intake frequency of foods, such as rice and bread, fish, meat, vegetables (non-juice), milk and yogurt and lactobacillus drinks, and inversely associated with a low intake frequency of vegetable and fruit juices. The associations were predominantly observed in women, and were independent of lifestyle-related and disaster-related factors.

\section{Author affiliations}

${ }^{1}$ Department of Epidemiology, Fukushima Medical University School of Medicine, Fukushima, Japan

${ }^{2}$ Radiation Medical Science Center for the Fukushima Health Management Survey, Fukushima, Japan

${ }^{3}$ Department of Public Health, Fukushima Medical University School of Medicine, Fukushima, Japan

${ }^{4}$ Department of Radiation Health Management, Fukushima Medical University School of Medicine, Fukushima, Japan

${ }^{5}$ Department of Disaster Psychiatry, Fukushima Medical University School of Medicine, Fukushima, Japan

${ }^{6}$ National Institute of Mental Health, National Center of Neurology and Psychiatry, Tokyo, Japan

${ }^{7}$ Department of Neuropsychiatry, Fukushima Medical University School of Medicine, Fukushima, Japan

Acknowledgements The authors would like to express their sincere appreciation for the support extended to them by the chairpersons, expert committee members, advisors and staff of the Fukushima Health Management Survey Group.

Contributors TO, SY, AO, MM, MH, NH, YS, HY, HT and MH were responsible for data collection and overseeing study procedures. MU and TO contributed to the design of this study. MU conducted the analysis and wrote the manuscript. SY, AO, MM, MH, NH, YS, HY, HT, MA, MN, HN, WZ and MH made significant contributions to the critical interpretation of the results in terms of important practical content. All authors read and approved the final version of the manuscript.

Funding This survey was conducted as part of Fukushima Prefecture's post-disaster recovery plans and was supported by the national 'Health Fund for Children and Adults Affected by the Nuclear Incident'.

Competing interests None declared.

Patient consent Obtained.

Provenance and peer review Not commissioned; externally peer reviewed.

Data sharing statement No additional data are available.

Open Access This is an Open Access article distributed in accordance with the Creative Commons Attribution Non Commercial (CC BY-NC 4.0) license, which permits others to distribute, remix, adapt, build upon this work noncommercially, and license their derivative works on different terms, provided the original work is properly cited and the use is non-commercial. See: http:// creativecommons.org/licenses/by-nc/4.0/

\section{REFERENCES}

1. Ohira T, Hosoya M, Yasumura S, et al., Fukushima Health Management Survey Group. Effect of evacuation on body weight after the Great East Japan Earthquake. Am J Prev Med 2016;50:553-60.

2. Satoh H, Ohira T, Hosoya M, et al. Evacuation after the Fukushima Daiichi nuclear power plant accident is a cause of diabetes: results from the Fukushima Health Management Survey. J Diabetes Res 2015;2015:627390.

3. Suzuki H, Ohira T, Takeishi $Y$, et al. Increased prevalence of atrial fibrillation after the Great East Japan Earthquake: results from the Fukushima Health Management Survey. Int J Cardiol 2015;198:102-5.

4. Yabe H, Suzuki $\mathrm{Y}$, Mashiko $\mathrm{H}$, et al., Mental Health Group of the Fukushima Health Management Survey. Psychological distress after the Great East Japan Earthquake and Fukushima Daiichi Nuclear Power Plant accident: results of a mental health and lifestyle survey 
through the Fukushima Health Management Survey in FY2011 and FY2012. Fukushima J Med Sci 2014;60:57-67.

5. Yasumura S, Hosoya M, Yamashita S, et al. Study protocol for the Fukushima Health Management Survey. J Epidemiol 2012;22:375-83.

6. Kawakami N. National survey of mental health measured by $\mathrm{K} 6$ and factors affecting mental health status (in Japanese). Research on Applied Use of Statistics and Information, Health Labour Sciences Research Grant 2006/2007.

7. Yokoyama Y, Otsuka K, Kawakami N, et al. Mental health and related factors after the Great East Japan earthquake and tsunami. PLOS ONE 2014:9:e102497.

8. Sakuma A, Takahashi Y, Ueda I, et al. Post-traumatic stress disorder and depression prevalence and associated risk factors among local disaster relief and reconstruction workers fourteen months after the Great East Japan Earthquake: a cross-sectional study. BMC Psychiatry 2015;15:58.

9. Lai JS, Hiles S, Bisquera A, et al. A systematic review and meta-analysis of dietary patterns and depression in community-dwelling adults. Am J Clin Nutr 2014;99:181-97.

10. Sanhueza C, Ryan L, Foxcroft DR. Diet and the risk of unipolar depression in adults: systematic review of cohort studies. J Hum Nutr Diet 2013;26:56-70.

11. Kessler RC, Barker PR, Colpe LJ, et al. Screening for serious mental illness in the general population. Arch Gen Psychiatry 2003;60:184-9.

12. Blanchard EB, Jones-Alexander J, Buckley TC, et al. Psychometric properties of the PTSD Checklist (PCL). Behav Res Ther 1996;34:669-73.

13. Furukawa TA, Kessler RC, Slade T, et al. The performance of the $\mathrm{K} 6$ and $\mathrm{K} 10$ screening scales for psychological distress in the Australian National Survey of Mental Health and Well-Being. Psychol Med 2003;33:357-62.

14. Sakurai $\mathrm{K}$, Nishi $\mathrm{A}$, Kondo $\mathrm{K}$, et al. Screening performance of K6/K10 and other screening instruments for mood and anxiety disorders in Japan. Psychiatry Clin Neurosci 2011:65:434-41.
15. Sauvaget $\mathrm{C}$, Allen $\mathrm{N}$, Hayashi $\mathrm{M}$, et al. Validation of a food frequency questionnaire in the Hiroshima/Nagasaki Life Span Study. $J$ Epidemiol 2002;12:394-401.

16. Nishi N, Yoshimura E, Ishikawa-Takata K, et al. Relationship of living conditions with dietary patterns among survivors of the great East Japan earthquake. J Epidemiol 2013;23:376-81.

17. Tsuboyama-Kasaoka N, Hoshi $\mathrm{Y}$, Onodera K, et al. What factors were important for dietary improvement in emergency shelters after the Great East Japan Earthquake? Asia Pac J Clin Nutr 2014;23:159-66.

18. Fujitani $\mathrm{K}$, Carroll M, Yanagisawa R, et al. Burnout and psychiatric distress in local caregivers two years after the 2011 Great East Japan Earthquake and Fukushima nuclear radiation disaster. Community Ment Health J 2016;52:39-45.

19. Jacka FN, Pasco JA, Mykletun A, et al. Association of Western and traditional diets with depression and anxiety in women. $A m ~ J$ Psychiatry 2010;167:305-11.

20. Jacka FN, Mykletun A, Berk M, et al. The association between habitual diet quality and the common mental disorders in community-dwelling adults: the Hordaland Health study. Psychosom Med 2011;73:483-90.

21. Gilbody S, Lightfoot T, Sheldon T. Is low folate a risk factor for depression? A meta-analysis and exploration of heterogeneity. $J$ Epidemiol Community Health 2007;61:631-7.

22. Kamphuis MH, Geerlings MI, Tijhuis MA, et al. Depression and cardiovascular mortality: a role for $n-3$ fatty acids? Am J Clin Nutr 2006;84:1513-17.

23. Mohammadi AA, Jazayeri S, Khosravi-Darani K, et al. The effects of probiotics on mental health and hypothalamic-pituitary-adrenal axis: a randomized, double-blind, placebo-controlled trial in petrochemical workers. Nutr Neurosci 2015. Published Online First: 16 Apr 2015. doi:10.1179/1476830515Y.0000000023

24. Nanri A, Kimura Y, Matsushita Y, et al. Dietary patterns and depressive symptoms among Japanese men and women. Eur J Clin Nutr 2010;64:832-9.

25. Gangwisch JE, Hale L, Garcia L, et al. High glycemic index diet as a risk factor for depression: analyses from the Women's Health Initiative. Am J Clin Nutr 2015;102:454-63. 